UNITED STATES

DEPARTMENT OF THE INTERIOR

GEOLOGICAL SURVEY

\title{
MULTICHANNEL SEISMIC REFLECTION DATA ACQUIRED \\ ON THE M/V CECIL H. GREEN IN THE GULF OF ALASKA, JUNE - AUGUST 1975 \\ By
}

Terry R. Bruns and Kenneth Bayer

OPEN-FILE REPORT

77-352

This report is preliminary and has not been edited or reviewed for conformity with Geological Survey standards and nomenclature. 
MULTICHANNEL SEISMIC REFLECTION DATA ACQUIRED ON THE M/V CECIL H. GREEN IN THE GULF OF ALASKA, JUNE - AUGUST 1975

Terry R. Bruns and Kenneth Bayer

\section{Introduction}

During June - August, 1975, the U.S. Geological Survey contracted with Geophysical Service, Inc., party 2942 aboard the M/V CECII H. GREEN, to acquire approximately $5500 \mathrm{~km}$ of 24 - and 48 -fold seismic reflection data in the Gulf of Alaska, roughly between Unimak Island at the end of the Alaska peninsula and Cross Sound east of Juneau, Alaska. Tracklines are shown in Figures 1 thru 5. Table 1 shows line numbers, shotpoint numbers, fold of coverage, field magnetic tape reel numbers, and final-stack magnetic tape reel numbers. In addition, high resolution records obtained with a Teledyne Model 405 mini-sparker system were acquired on some of the lines and are indicated in Table 2 .

\section{Seismic recording system}

(1) Amplifier and Magnetic Recorder:

DFS IV digital instrument, with a dynamic range of $81 \mathrm{db}$ were used to record 48-trace, 24- and 48-fold seismic data on one inch 21-track magnetic tape at $712 \mathrm{bpi}$. The sample rate was 4 milliseconds. Field filters had a high cut of $62 \mathrm{~Hz}$ with a $72 \mathrm{db} /$ oct slope, and a low cut of $8 \mathrm{~Hz}$ with an $18 \mathrm{db} /$ Oct slope. Record length was primarily $6 \mathrm{sec}$; longer record times were used in deep water. System noise was less than $0.25 \mu \mathrm{v}$.

(2) Streamer:

The GSI 2400-m streamer used 48 live sections containing 30 acceleration-cancelling geophones per section. Streamer depth was maintained 
at approximately $14 \mathrm{~m}$ except in shallow water. Depth was controlled by weighting the streamer to approximately neutral buoyancy, by use of nine depth-control birds strategically located along the streamer, and to a minor extent by ship speed and length of lead-in. Streamer geometry is shown in Figure 6. Geophone group geometry and hydrophone response are shown in Figure 7.

(3) Energy Source:

The energy source for the survey was a tuned $1200 \mathrm{cu}$. in. airgun array towed $26 \mathrm{~m}$ off the stern at a depth of $10 \mathrm{~m}$. The distance from the satellite antenna to the center of the airgun array during this survey was $54 \mathrm{~m}$. Air pressure was maintained at 1600-2000 psi by two compressors. Guns were fired at $25 \mathrm{~m}$ or $50 \mathrm{~m}$ intervals depending on fold of coverage. The airgun array geometry is shown in Figure 8. Figure 9 shows the approximate form and frequency spectrum of the resulting pulse.

\section{Navigation}

Shotpoints were located by an integrated 980A Geonav survey system utilizing doppler sonar dead-reckoning control with Loran $\mathrm{C}$ as a secondary system. Absolute satellite positions were taken on the average of every 1.5 hours. Betwenn fixes, ship velocities were monitored continuously with a doppler sonar or a Loran C 980-A interface operated by offshore Navigaton, Inc.

\section{Processing}

Data were processed by Petty-Ray Geophysical, Division of Geosource, Inc., in Houston, Texas. The processing sequence for each line is shown on the stacked sections; an example is shown in Figure 10. The stacked sections were filmed at $21 / 2 \mathrm{H} / \mathrm{sec}$ and $20 \mathrm{traces} / \mathrm{inch}$; an example of a final stacked section is shown in Figure 11. 


\section{Available Data}

Final shotpoint maps are available at two scales: $1: 250,000$ and $1: 96,000$ ( 1 in $=8000 \mathrm{ft}$ ), and a map index is shown in Figure 12. Shotpoint locations on the maps are at the antenna position. For reference purposes, a cruise identifier code, EGAL-75-XB is annotated on all reports and logs associated with the data.

The data for this survey are available from:

Marine Technical Data Center

345 Middlefield Road

Menlo Park, CA 94205

Phone: (415) 323-8111, ext. 2074

and

National Geophysical and

Solar Terrestrial Data Center (NGSDC)

EDS/NOAA; BOulder, CO 80302

Data available from NGSDC only:

Sepia or paper print of full-scale final stack seismic sections.

Sepia or paper print of corrected velocity gathers.

Full-scale final shotpoint maps at scales of $1: 250,000$ and 1:96,000.

Magnetic tape of shotpoint locations.

Magnetic Tape of bathymetry.

Data available from Marine Technical Data Center:

Microfilm of final stack seismic sections.

Microfilm of the 1:250,000 shotpoint maps.

Data available from both NGSDC and Marine Technical Data Center:

Microfilm of observers logs.

Microfilm of velocity analyses.

Microfilm of single trace sections.

Microfilm of corrected velocity gathers.

Map Index Sheet.

Microfilm of high-resolution seismic reflection data.

Microfilm copies of final reports from GSI, ONI, and Petty Ray. 


\section{FIGURE CAPTIONS}

Figure 1: Trackline Map 1: Cross Sound to Icy Bay, Eastern Gulf of Alaska

Figure 2: Trackline Map 2: Icy Bay to Kayak Island, Eastern Gulf of Alaska

Figure 3: Trackline Map 3: Kayak Island to Middleton Island, Eastern Gulf of Alaska

Figure 4: Trackline Map 4: Middleton Island to Chirikof Island, Kodiak Shelf, Western Gulf of Alaska

Figure 5: Trackline Map 5: Chirikof Island to Unimak Island, Shumagin Shelf Western Gulf of Alaska

Figure 6: Streamer Geometry

Figure 7: Geophone Group Geometry and Hydrophone Response.

Figure 8: Airgun Array Geometry

Figure 9: Airgun Pulse Form and Frequency Spectrum

Figure 10: Example of processing sequence for line 406 (See Fig. 11)

Figure 11: Part of the final stacked section for Line 406 near Icy Bay, Eastern Gulf of Alaska

Figure 12: Index map for $1: 250,000$ and 1:96,000 final shotpoint location map. 
TABLE 1: Line and reel listing for multichannel seismic reflection data acquired on the M/V CECIL H. GREEN in the Gulf of Alaska, June-August 1975.

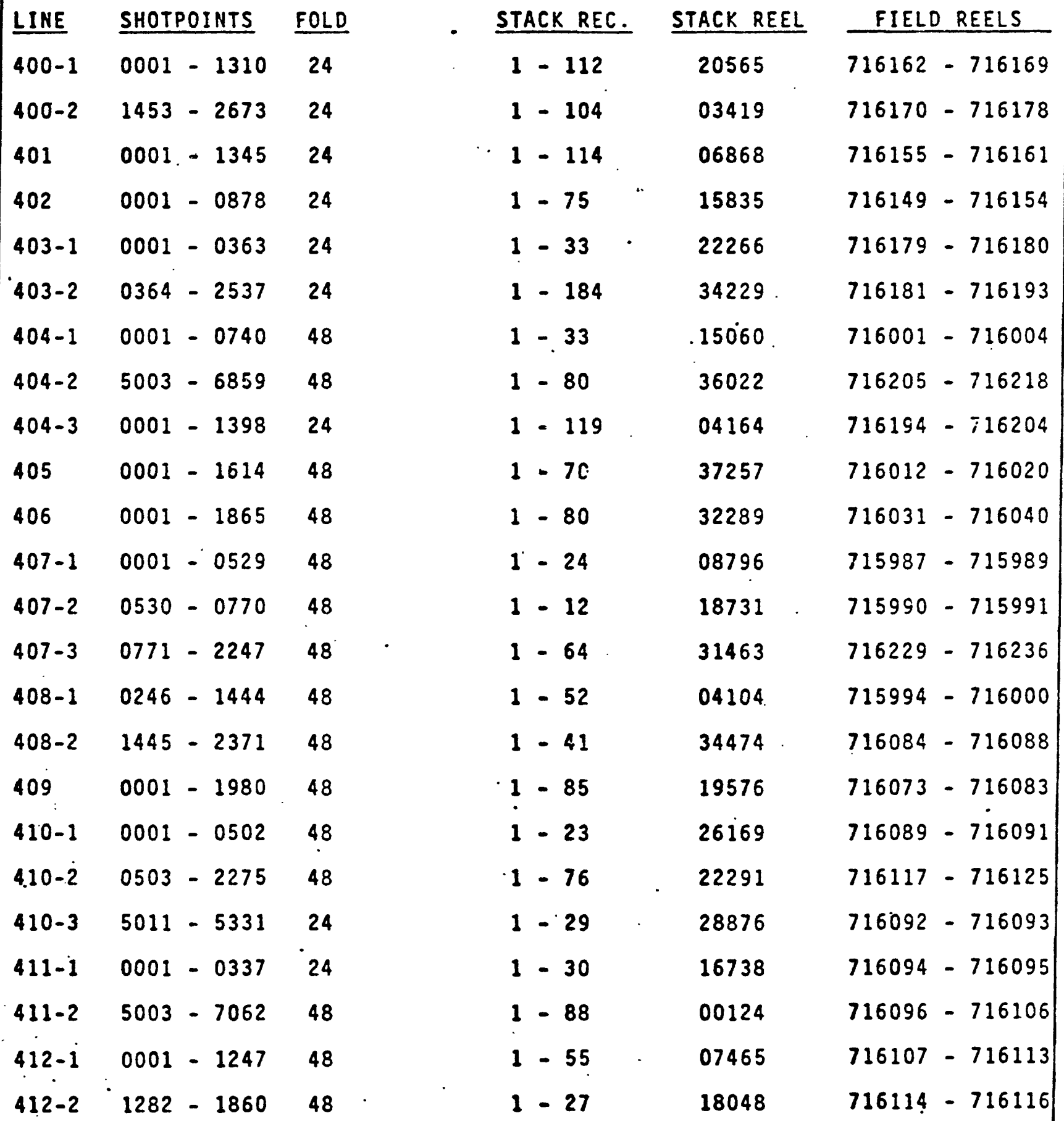


LINE SHOTPOINTS SOLD

413-3 $0001-0220 \quad 48$

$413-1 \quad 0221-0960 \quad 48$

$413-2 \quad 1361-2371 \quad 48$

414-1 $1301-2767 \quad 48$

414-2 $0001 \cdot 0784 \quad 48$

$415 \quad 0001-1180 \quad 48$

$416 \quad 0001-0993 \quad 48$

$417 \quad 0001-1852 \quad 48$

$418 \quad 0001-0816 \quad 48$

419-1 $0001-0169 \quad 48$

$419-2 \quad 0162-1502 \quad 48$

$420 \quad 0001-2275 \quad 48$

$421-1 \quad 0191-0580 \quad 48$

421-2 $1002-345 i \quad 48$

$4220001-3023 \quad 48$

423-1 $0001-0202 \quad 48$

423-2 $0405-3175 \quad 48$

424-1 $0001-0551 \quad 48$

424-2 $0771-2738 \quad 48$

425-1 $0001-365 j \quad 48$

425-2 5001- $6561 \quad 24$

$426 \cdot 0001-2237 \quad 48$

$427 \quad 0001-166648$

428-1 $0200-0910 \quad 48$

428-2 $0911-2036 \quad 48$

428-3 $2037-2446 \quad 24$

$429 \quad 0001-1749 \quad 48$
STACK REC. STACK REEL

1 - 33

$1-45$

$1-12$

$1-64$

$1-35$

1 - 52

$1-44$

$1-80$

$1-36$

$1-9$

1.- 58

1 - 97

$1-19$

$1-105$

1 - 128

$1-11$

. -137

$1-119$

$1 \cdot 119$

$1-155$

1 - 132

$1-96$

$1-72$

1 - 32

$1-49$

1 - 37

$1-75$
35652

38437

33158

30023

16393

09140

06359

37536

29683

21535

14462

18649

04656

37410

08143

13624

13624

38574

38574

23006

31612

08668

31137

10562

07412

06215

02474
FIELD REELS

715959 - 715960

715961 - 715964

$716054-716059$

$716060-716069$

$715955-715958$

$715889-715894$

$715884-715888$

$715874-715883$

$716005-716009$

$715645-715645$

$715646^{\circ}-715.652$

$715633-715644$

$715857-715858$

$715861-715873$

$715604-715619$

$715586-715587$

$715589-715603$

715571 - 715573

$715575-715584$

$715825-715843$

$716280-716293$

$715812-715824$

$715735-715743$

$715721-715724$

$715758-715764$

$716294-716297$

$715706-715714$ 
LINE SHOTPOINTS FOLD

430-1 $0001-106848$

$430-2 \quad 1445-1876 \quad 48$

$4310466-117324$

$4 \dot{32} \quad 0001-1259 \quad 48$

450-1 $0001-0479^{\circ} 48$

$450-2 \quad 2016-391148$

451-1 $0046-0722 \quad 48$

451-2 $1856-2435 \quad 48$

451-4 $3252-4025 \quad 48$

452-1 0001 - $0429 \quad 48$

$452-2 \quad 1076-536348$

453-1 $0001-079024$

453-2 $0791-144124$

$4540001-2483 \quad 48$

455-1 $0001-2500 \quad 48$

455-2 2506-4033 48

455-3 $4311-8285 \quad 48$

$\begin{array}{llll}456 & 0001-4033 \quad 48\end{array}$

457-1 $0001-0334 \quad 48$

457-2 $0545-1700 \quad 48$

457-3 $1842-399148$

$458 \quad 0001-240948$

$459 \quad 0001-3279 \quad 48$

$460 \quad 0001-4259 \quad 48$

$\begin{array}{lll}461 & 0001-2109 \quad 48\end{array}$

462-1 $0001-038548$

$462-20386-203148$
STACK REC. STACK REEL

$1-47$

34653

$1-20$

1. 61

$1-55$

$1-22$

$1-81$

$1-31$

$1-27$

$1-35$

1 - 20

$1 \cdot-181$

1. -68

$1: 57$

1 - 106

1. 107

1. 66

1 - 168

1 - 171

$1-16$

1 - 51

1- 92

$1-103$

1. 139

1. 180

$1-90$

$1-.19$

1 - 71
17914

18102

.02576

32586

20446

35961

16821

32444

16829

38405

25327

34949

07672

01076

11844

09048

31919

30074

23528

34527

08186

06876

08471

05828

23221

00246
FIELD REELS 715700 - 715705

$715716-715718$

$716259-716262$

716240 - 716246

$716041-716043$

$716219-716228$

$716021-716024$

716044 - 716046

716251 - 716254

$715916-715918$

715922 - 715944

715255 - 716258

716263 - 716266

$716134-716148$

715620 - 715632

715945 - 715953

715967 - 715986

715895 - 715915

$715725-715726$

$715728-715733$

$715845-715855$

$715799-715811$

$715672-715688$

715776 - 715798

715689 - 715699

$715765-715766$

$715767-715775$ 
LINE SHOTPOINTS FOLD

$4630001-0803 \quad 48$

$4640001-1861^{\circ} 48$

$465^{\circ} 0001-1777 \quad 48$

$466 \quad 0001-1657 \quad 48$

$467 \quad 0001-1788 \quad 24$

$468 \quad 0001-0655 \quad 48$

500-1 $0001-1104 \quad 24$

$500-2 \quad 1471-1580 \quad 24$

$500-3 \quad 1811-2310 \quad 24$

$500-4 \quad 3415-4010 \quad 24$

$500-5 \quad 4102-4672 \quad 24$

$500-6 \quad 5170-6650 \quad 24$

500A-4 $0001-0790 \quad 24$

500A-5 $0791-2216 \quad 24$

500A-6 $2217-2766 \quad 24$

500A-7 $2767-3361 \quad 24$

$501 \quad 0001-011024$

501-1 $0115-150024$

501-2 $1501-28[]$.

501-3 $2801-3900$. 24

$501-4 \quad 3901-4799 \quad 24$

$502 \quad 0001-140124$

503-1 $0001-051124$

503-2 $0512-1405 \quad 24$

504-1 $0970-139124$

504-2 $2200-2498 \quad 24$

504-2CT.2508 - $2728 \quad 24$
STACK REC. STACK REEL

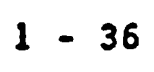

30940

$1-80$

37308

$1-76$.

09978

$1-72$

17049

$1-151$

19211

$1-30$

$1-94$

34868

04855

$1-12$

$1-44$

1- 52

$1-50$

$1-126$.

1. 68

$1-121$

$1-48$

$1-52$

$1-11$

1 - 118

1 - 111

1 - 94

$1-77$

$1-119$

1 - 45

1 - 77

1 - 58

1 - 27

$1-21$
19588

15585

38673

31229

17936

31301

27838

18096

06565

15421

2377.

36151

27865

11310

32459

23053

12714

12509

00085

00085
FIELD REELS

$715744-715747$

$715748-715757$

715662 - 715671

715653 - 715661

716267 - 716279

$716247-716250$

$715490-715495$

$715498-715498$

$715500 \cdot 715502$

715540 - 715543

$715545-715548$

$715550-715557$

$716298-716301$

$716310-716316$

$716332-715334$

$716335-716339$

$715428-715428$

$715430-715438$

$715446 \cdot 715452$

$715462-715467$

715477 - 715481

$715405-715412$

$716302-716304$

716305 - 716309

$715563-715566$

$716318-716320$

$716330-716331$ 
LINE SHOTPOINTS FOLD

$505 \quad 0693-2668 \quad 24$

505-1 $0001-012024$

506-1 $0001-115624$

506-2 $1230-185624$

$507 \quad 0001-157524$

$508 \quad 0001-1750 \quad 24$

$509 \quad 0001-162524$

$510 \quad 0001-1002 \quad 24$

5i1 $0001-2138 \quad 24$

$512 \quad .0001-0609.24$

$513 \quad 0001-151824$

$5140001-110924$

515. $0004-078024$

$5160811-144224$

$5170001-1270.24$

\begin{tabular}{ll}
$521 \quad 0001-1836 \quad 24$ \\
\hline
\end{tabular}

$5220001-170024$

$\begin{array}{lll}530 & 0002-1057 \quad 24\end{array}$

$531 \quad 0001-1200 \quad 24$

$5320001-142824$

$533 \quad 0001-208324$
STACK REC. STACK REEL $1-167$

$1-12$

$1-99$

$1-55$

$1 \cdot 134$

1 - 148

$1-138$

$1-86$

$1-181$

$1-53$

$1-129$

$1-95$

1 - 67

$1-55$

$1-108$

$1-155$

1 - 144

1 - 90

1 - 102

1 - 121

$1-176$
00046

01242

34555

32350

16992

25258

29958

05849

01213

02954

28816

14154

36424

03115

22403

23837

21152

30218

03870

34994

15434
FIELO REELS

$715514-715526$

715509 - 715509

$715503-715508$

$715528-715532$

$715482-715489$

$715468-715476$

$715453-715461$

$715439-715445$

$715413-715426$

715383 - 715386

715374 - 715382

715393 - 715399

$715388-715392$

$715400-715404$

716321 - 716329

715350 - 715363

715364 - 715373

$715330-715336$

$715313-715320$

715321 - 715329

715337 - 715349 
Table 2 : Line and shotpoint listing for high resolution seismic reflection data acquired on the $\mathrm{M} / \mathrm{V}$ Cecil $\mathrm{H}$. Green in the Gulf of Alaska, June- August 1975.

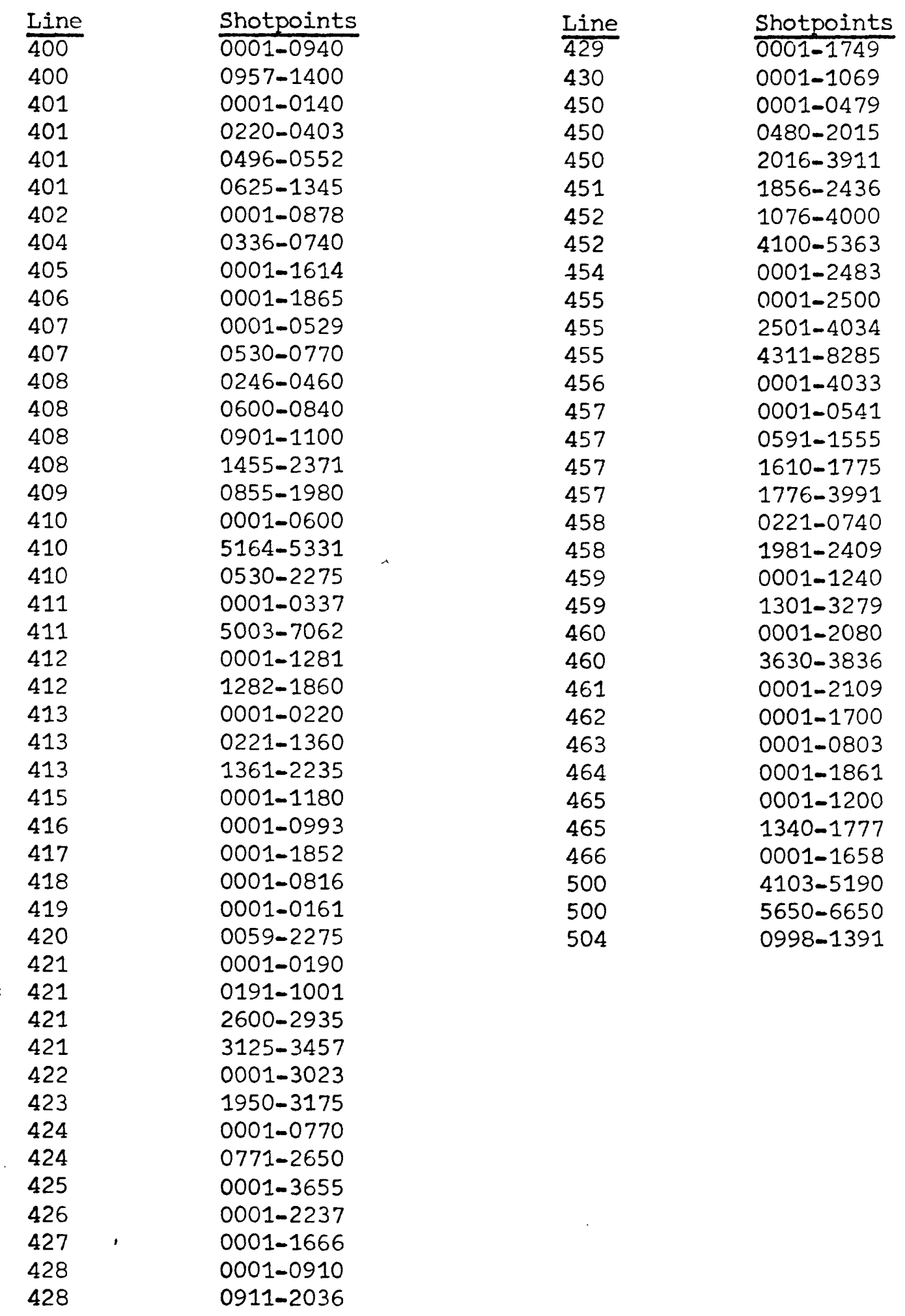




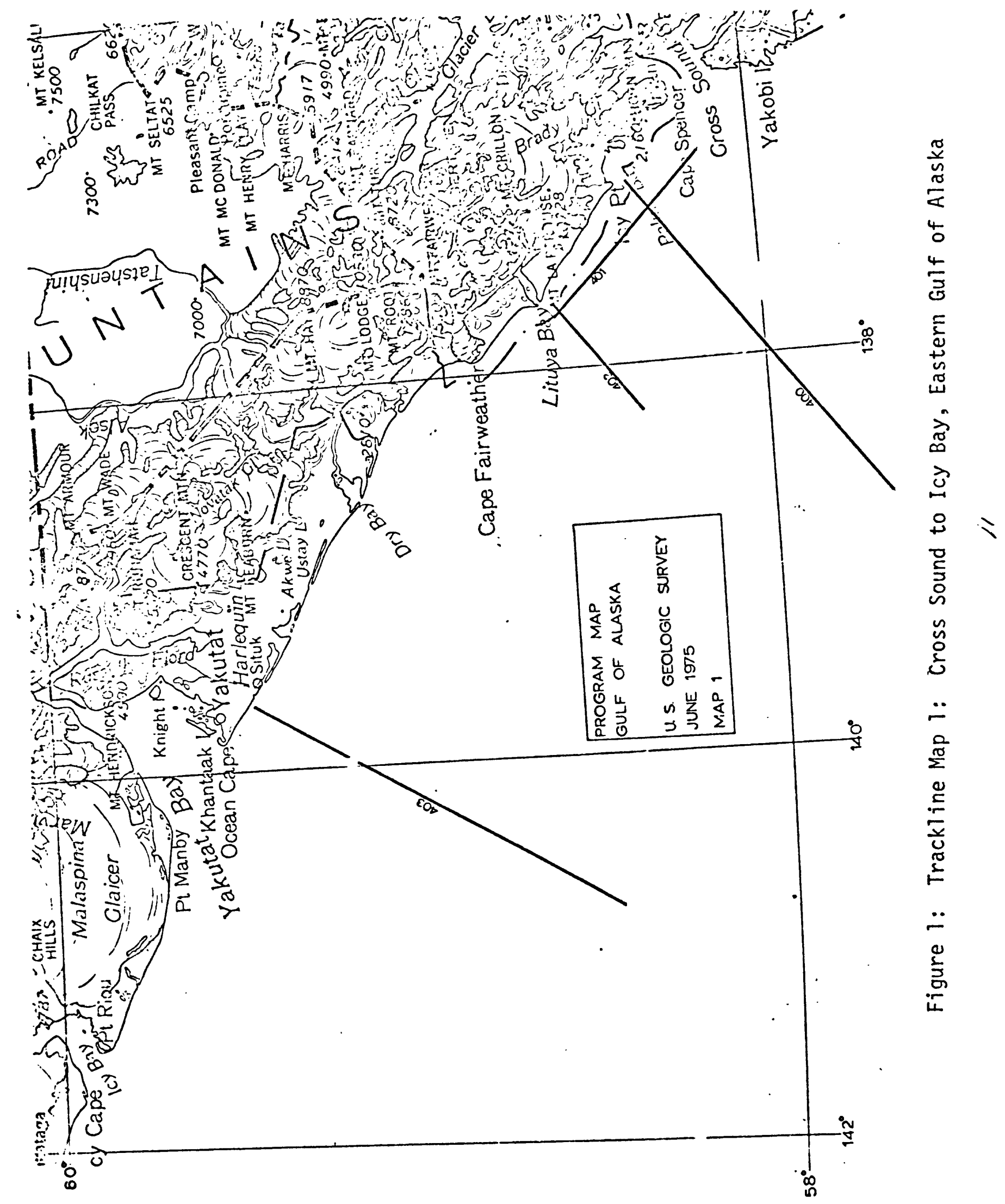




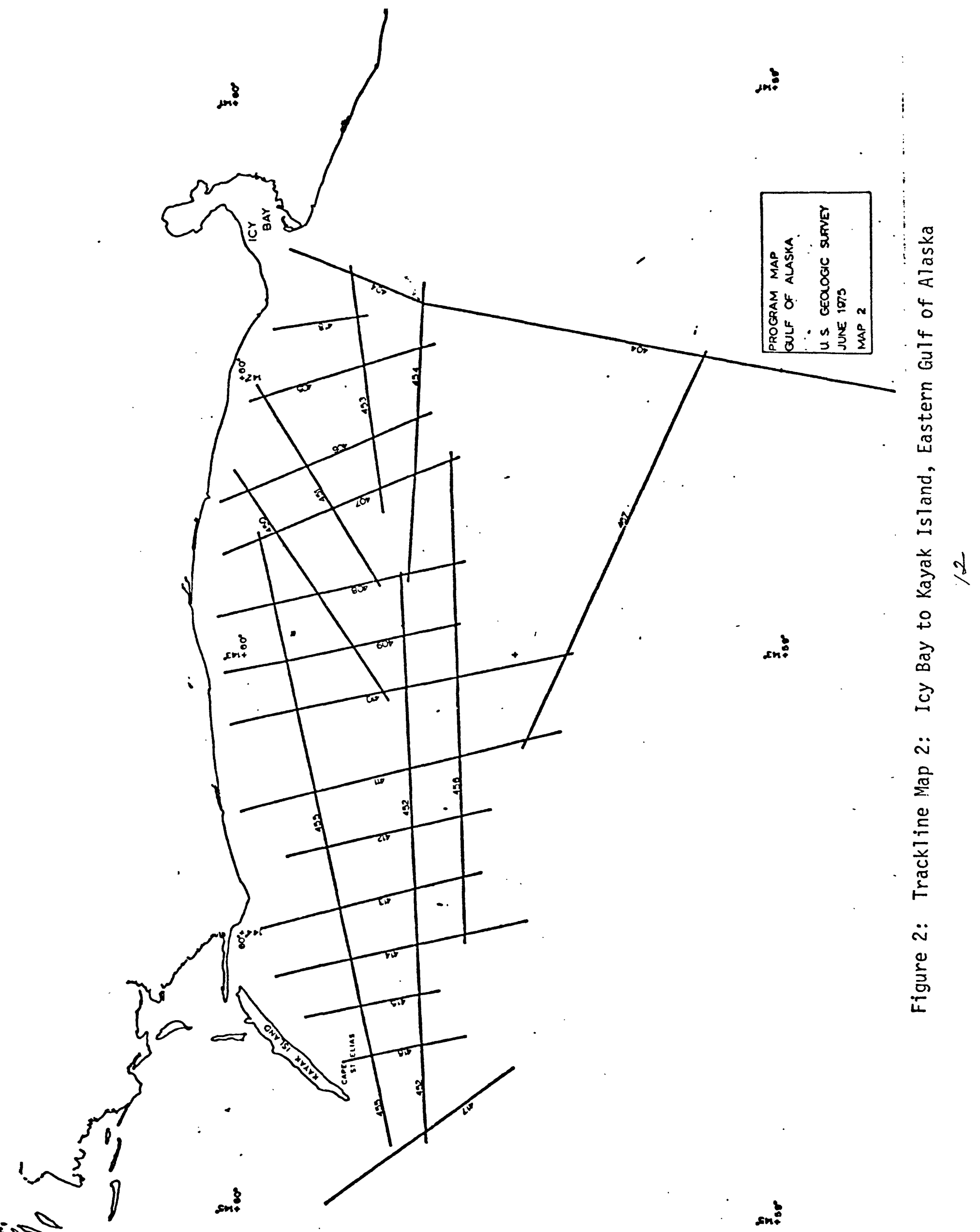




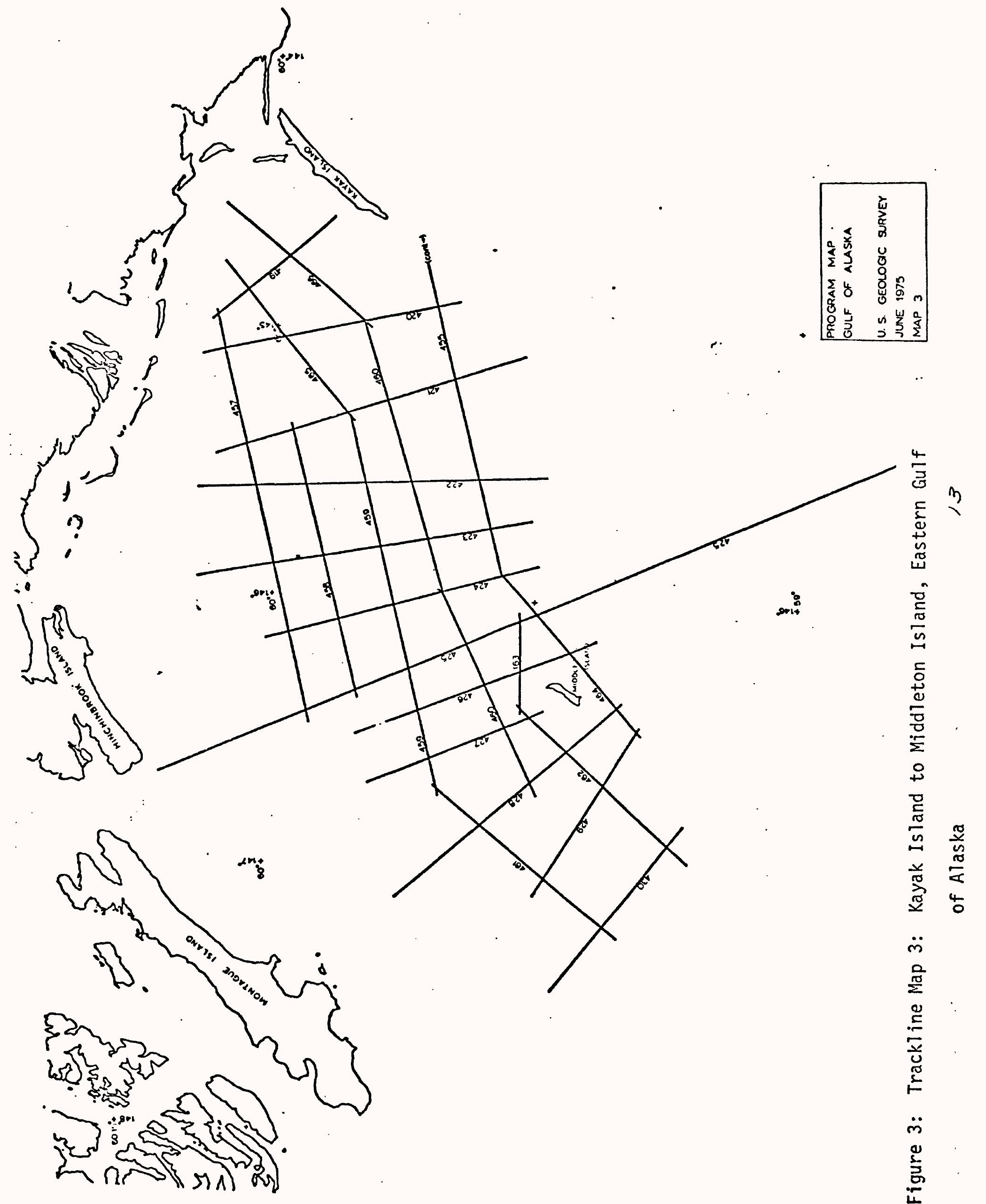




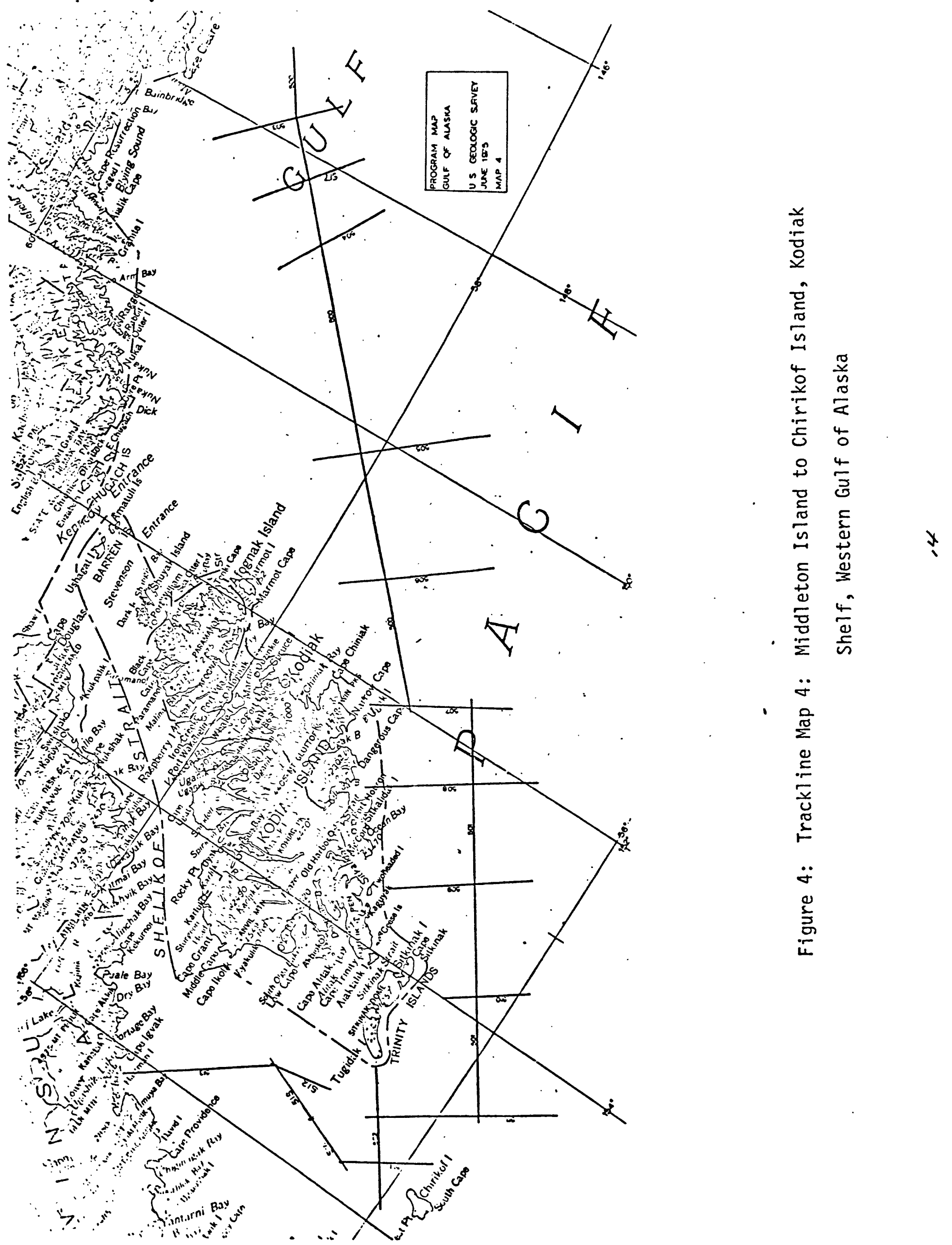




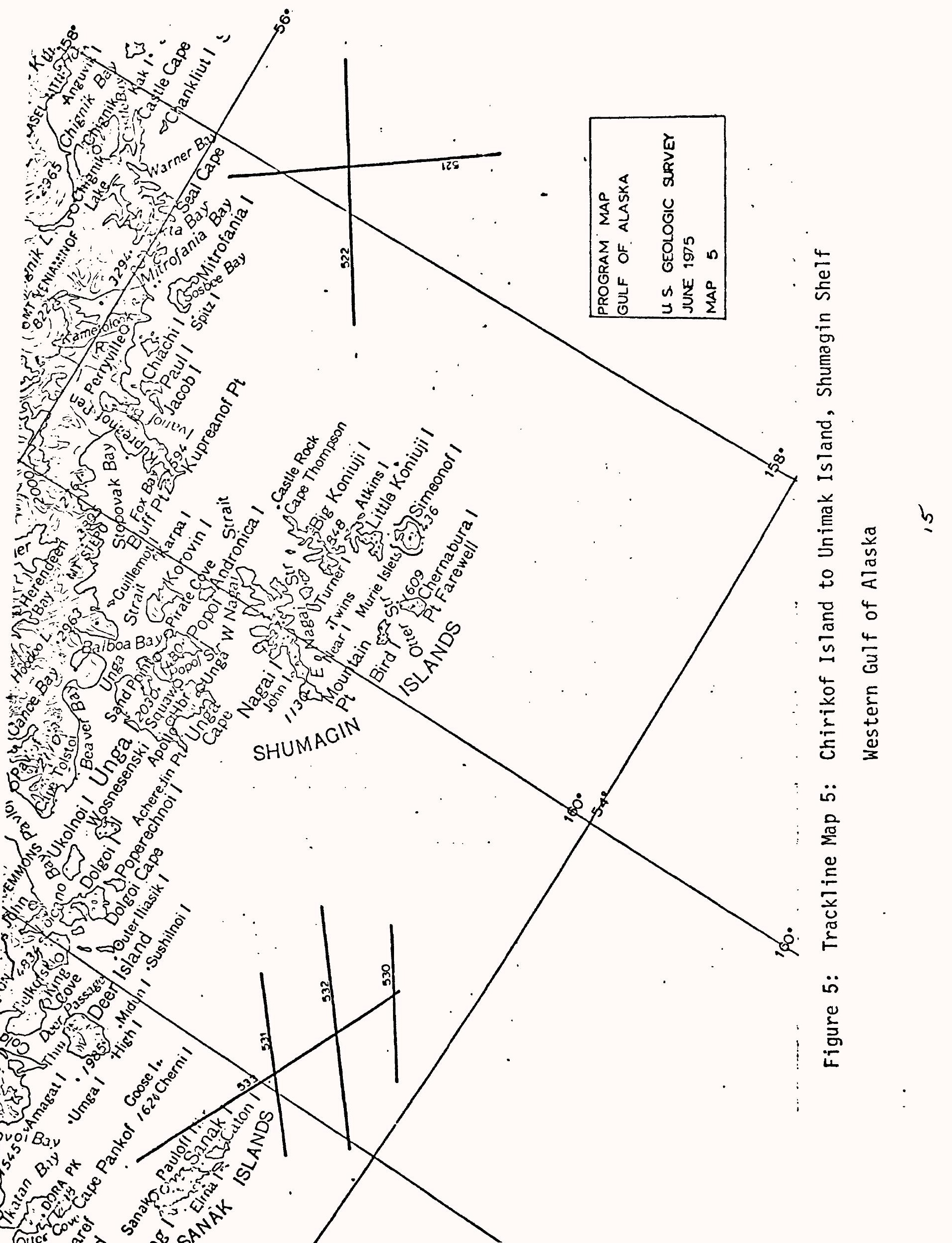




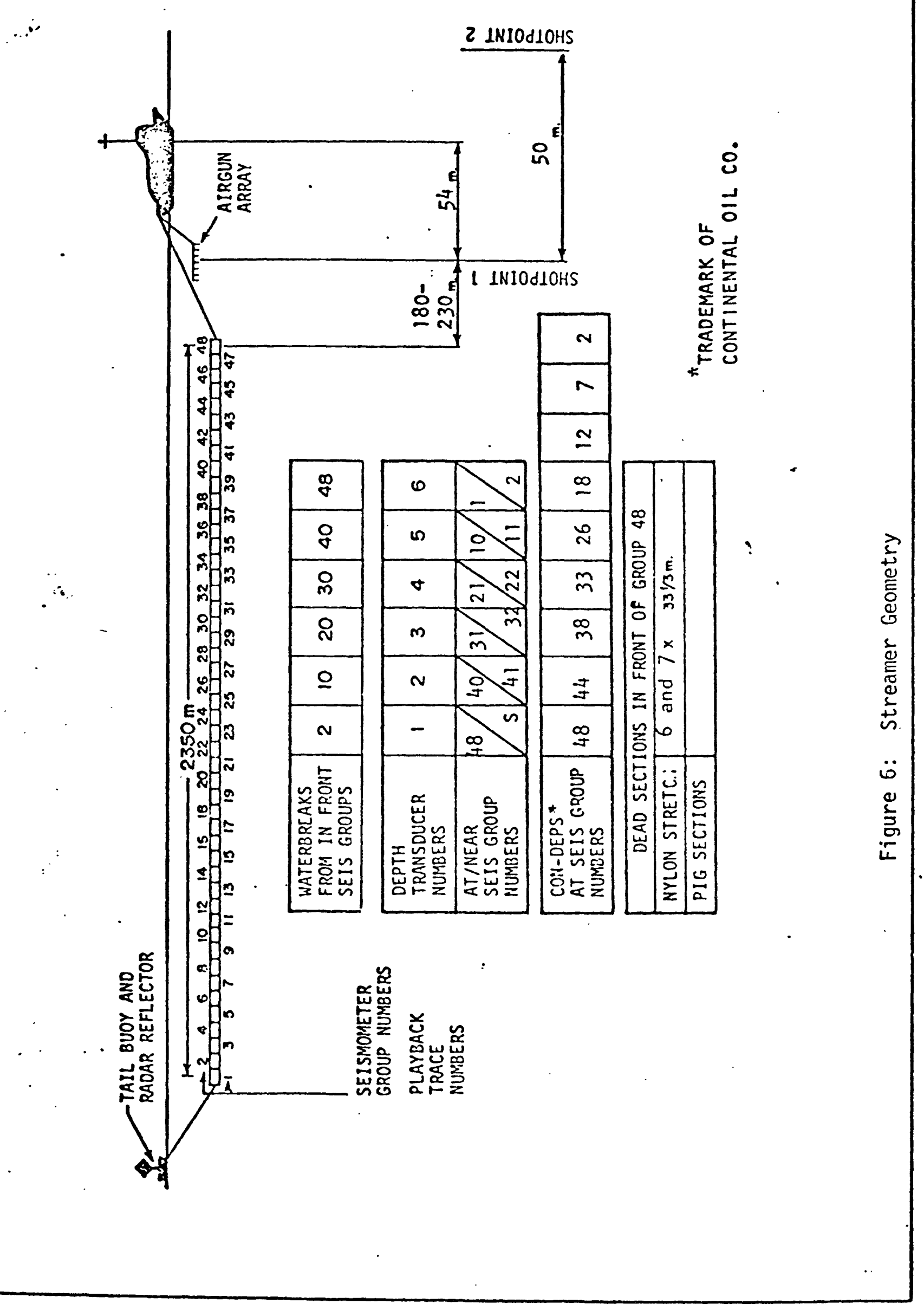


STREAMER RESPONSE

ACCELERATION-CANCELLING HYDROPHONE

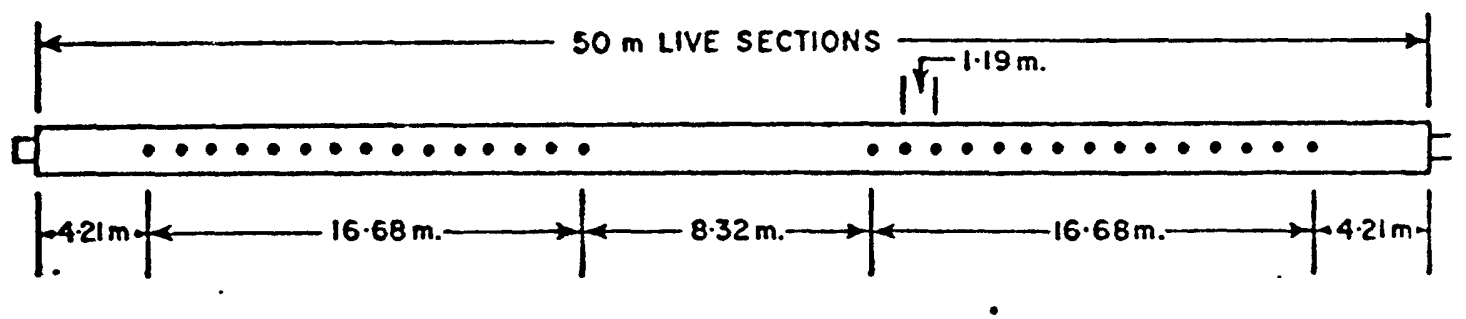

- 30 ACCELERATION-CANCELLING HYDROPHONES PER 50-M SECTION

- EACH HYDROPHONE CONTAINS THO CRYSTAL TUBES (approx. $0.96-$ in.

OD $\times 0.75-i n$. ID $\times 0.22-i n$.

in length) WIRED IN PARALLEL

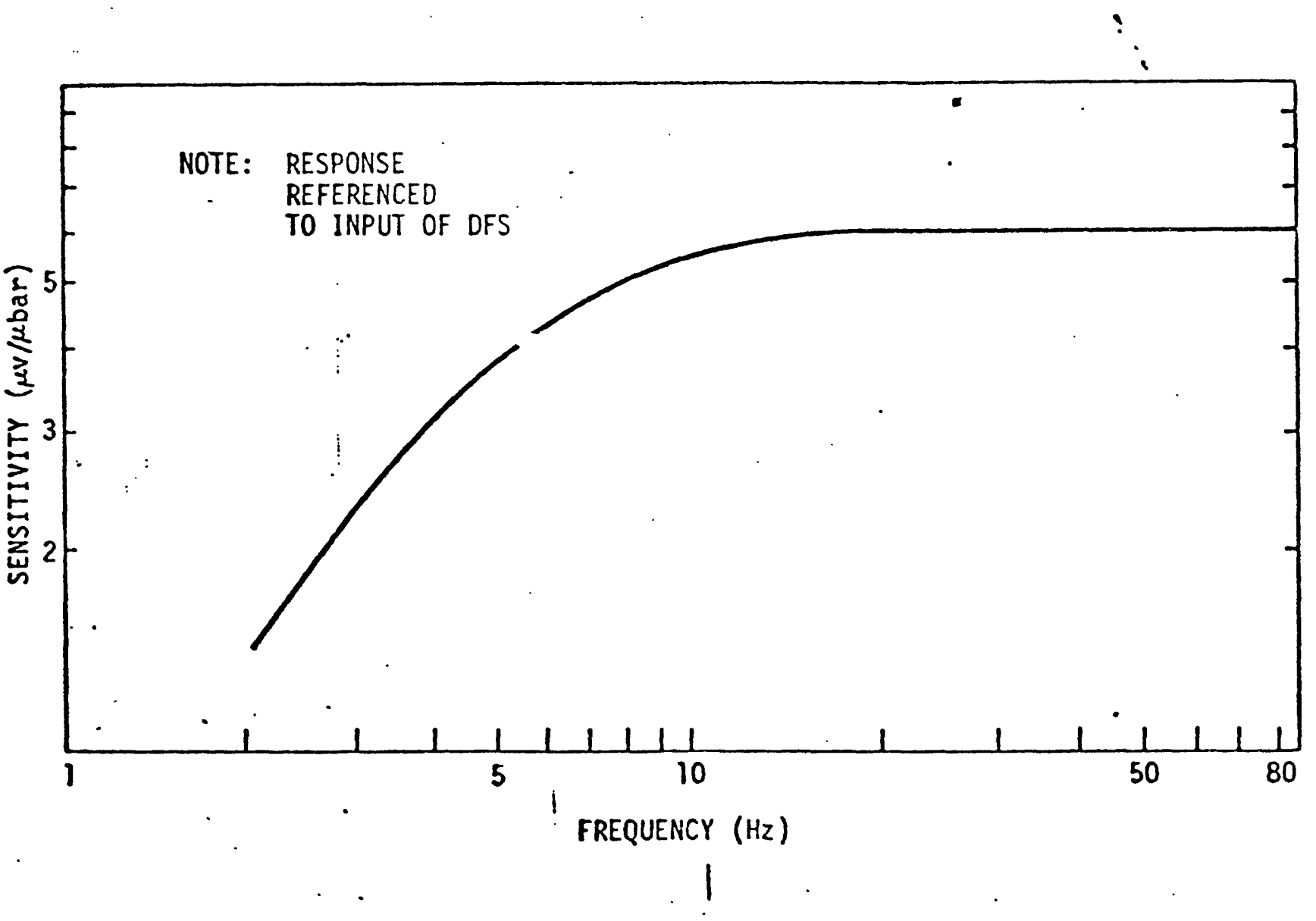

Figure 7: Geophone Group Geometry and Hydrophone Response. 


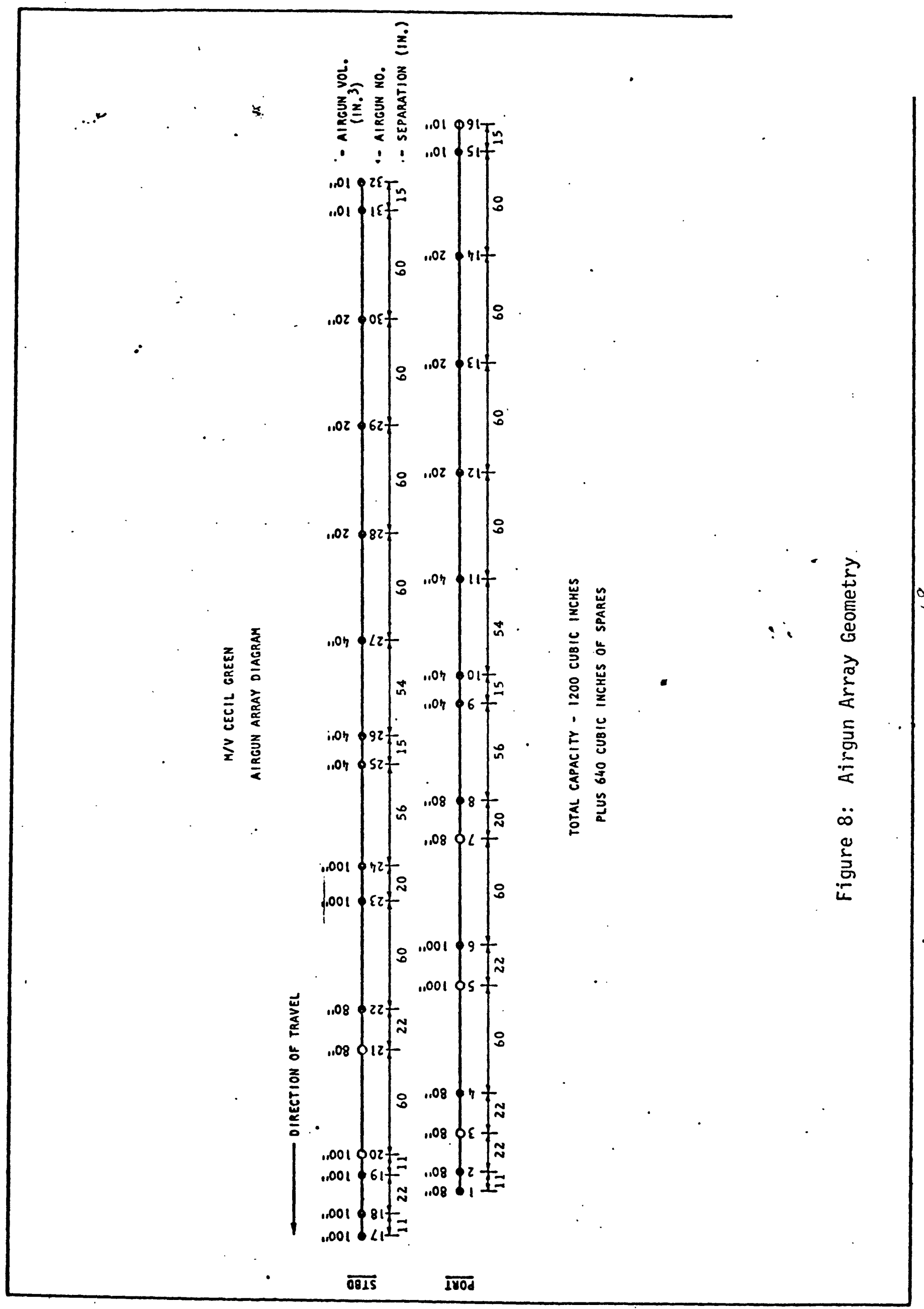




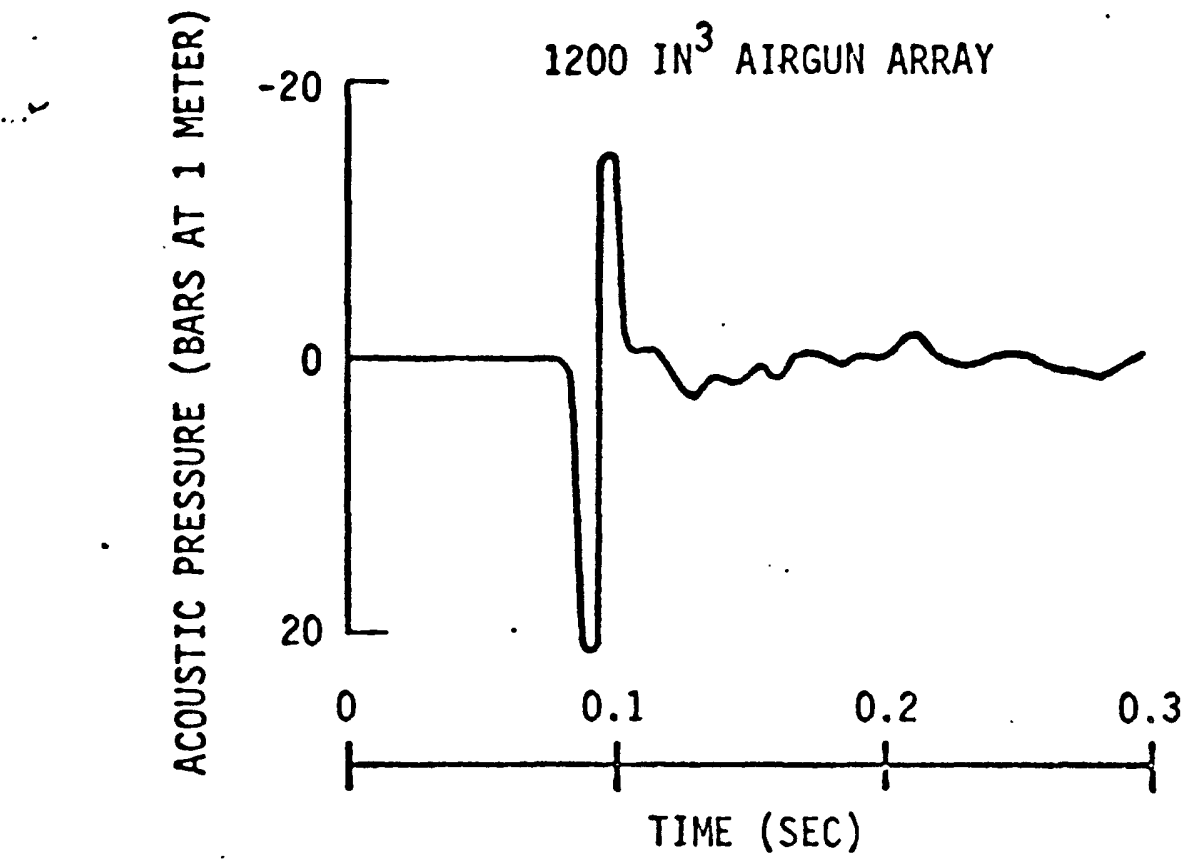

MEASURED PULSE FORM

FILTERED: OUT - $248 \mathrm{~Hz}$

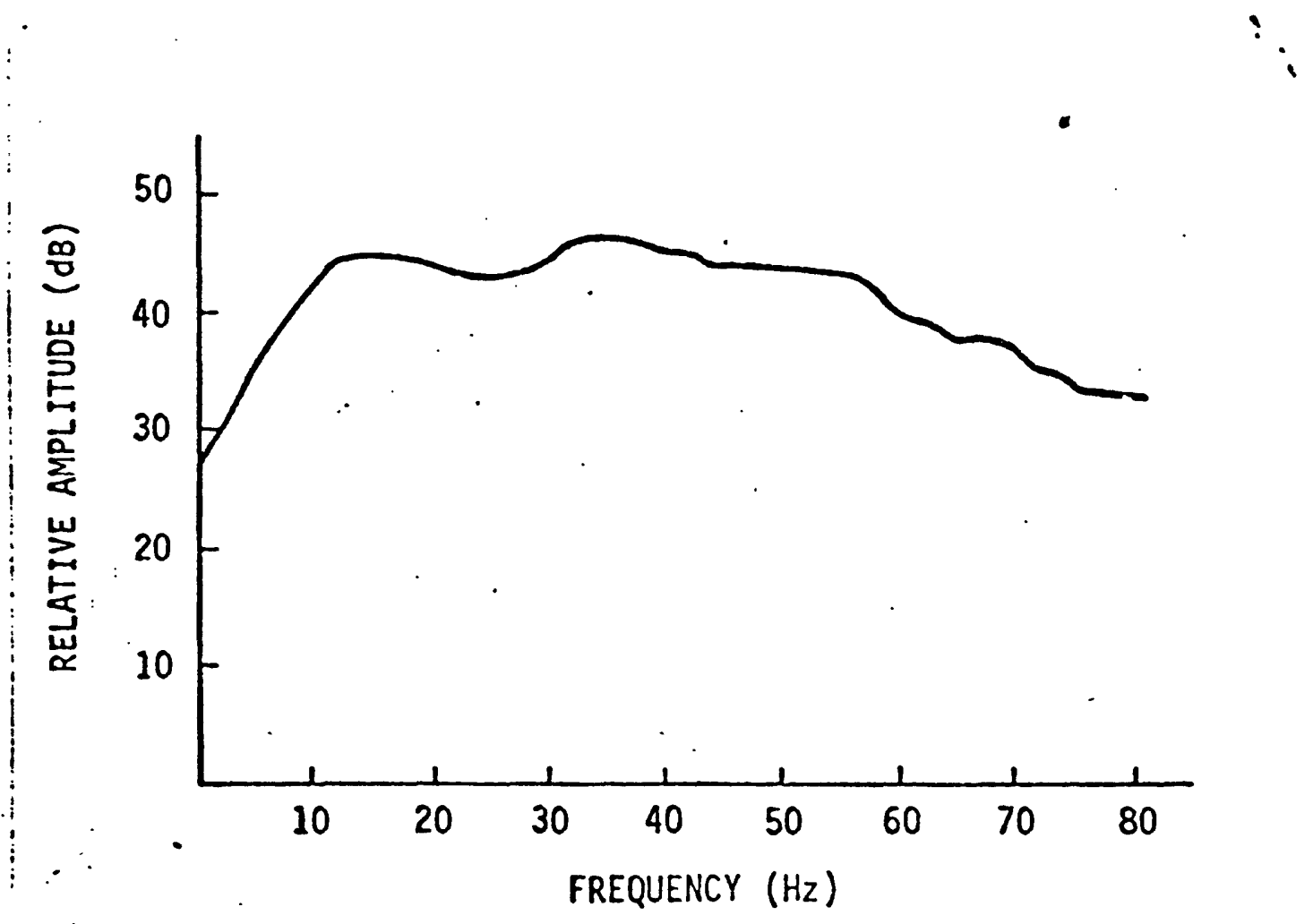

FREQUENCY SPECTRUM

Figure 9: Airgun Pulse Form and Frequency Spectrum 
U.S. GEOLOGICAL SURVEY

GULF OF ALASKA EGAL-75-XB

LINE 406

SP. 1 - 1865

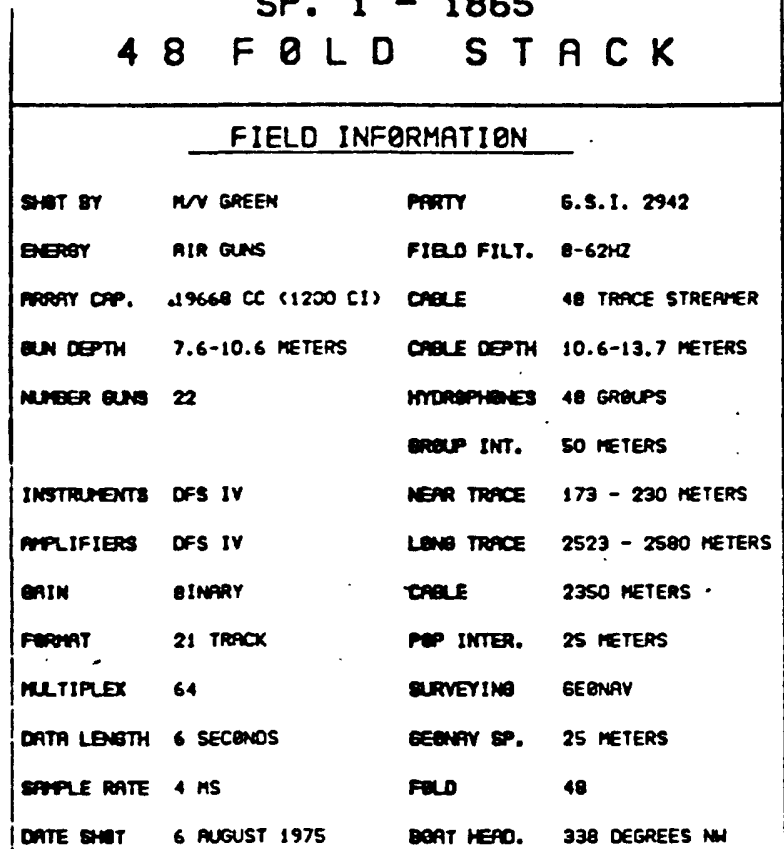

DATE ENT G RUGUST 1975 BOAT HEAD. 338 OESREES MH

PROCESSING SEQUENCE

Processing CEnter houstentexas

1) EOIT DEMLIIPLEX - PETTY-RAY MPLX. I FQRMAT

2) AMPLITUOE RECQVERY

3) OECONVELUTION 260 MS "SPIKE" WITH 2000 MS WINOQH HINDEH START - TR $1=1950 M S$ TR $48=300 M S$ HINOOH END - TR $I=2950 M S$ TR $48=2300 M S$

4) VELECITY ANALYYSIS (VELSTACK) AVERAGE 2.4 KILEMETERS 5) NBRMAR MOVEEUT CORRECTIENS RNO STATICS 12 MS S.L. DATUM

6) 40 FRD CEMMON DEPTH POINT STACK CORRECTED GATHERS EVERY 24 DP.

7) BANOPASS FREQUENCY FILTER "BRHSBY" FILTERS PRERATEO BETMEEN TIMES

\begin{tabular}{|c|c|c|c|c|}
\hline $\begin{array}{l}\text { START TIIRE } \\
0.000 \\
0.700 \\
1.500 \\
1.900 \\
2.500 \\
6.000\end{array}$ & & $\begin{array}{r}F 1 \\
7 \\
6 \\
5 \\
4 \\
4\end{array}$ & $\begin{array}{l}F 2 \\
14 \\
12 \\
10 \\
8 \\
8 \\
8\end{array}$ & $\begin{array}{l}\mathbf{F 3} \\
45 \\
35 \\
30 \\
25 \\
20 \\
20\end{array}$ \\
\hline
\end{tabular}

8) BRAANCE 5000-3000 DECAY HINOEH LENGTH 25ONS

9) CEMMENTS

MINDE. START TIME AND MUTE TIMES VARY WITH MATER DEPTH VELLCITIES PRERATED BETWEEN FUNCTIBNS

STATION LBCATIONS ARE GLN LOCATIONS

I KILQMETER S. C8 CENTIMETERS

WRTER DEPTHS $1031-18$

\section{Pelly-Roy}




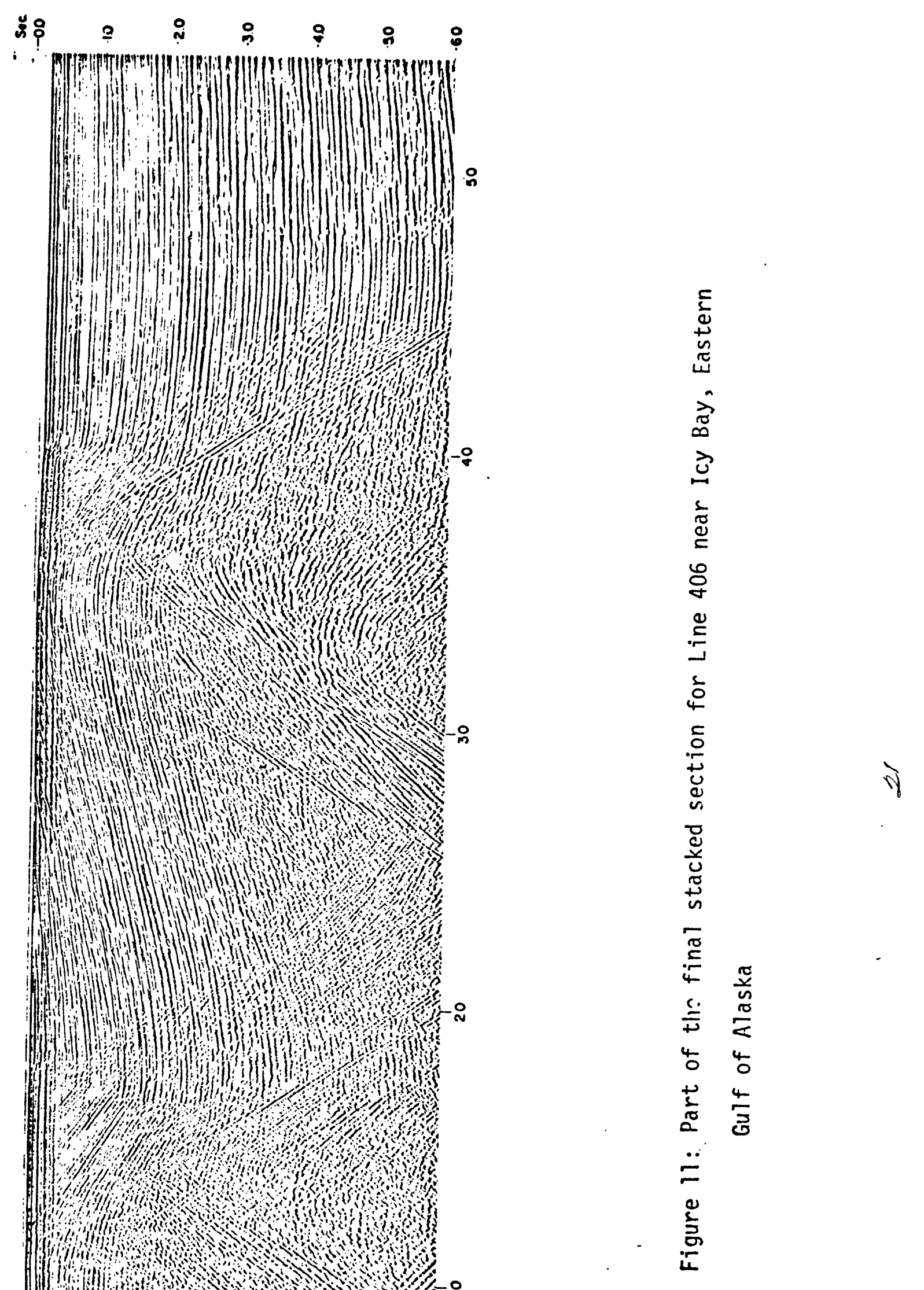


